\title{
White matter microstructure of attentional networks predicts attention and consciousness functional interactions
}

\author{
Abbreviated title: \\ Anatomical predictors of the interactions between attention and consciousness
}

Ana B. Chica ${ }^{1}$, Michel Thiebaut de Schotten ${ }^{2}$, Paolo Bartolomeo ${ }^{3}$, and Pedro M. Paz-

\section{Alonso $^{4}$}

1. Department of Experimental Psychology, and Brain, Mind, and Behavior Research Center (CIMCYC), University of Granada, Spain.

2. Brain Connectivity and Behaviour group, Frontlab, Institut du Cerveau et de la Moelle épinière (ICM), and CNRS U7225, Inserm U1127, GH Pitié-Salpêtrière.

3. INSERM U 1127, CNRS UMR 7225, Sorbonne Universités, and Université Pierre et Marie Curie-Paris 6, UMR S 1127, Institut du Cerveau et de la Moelle épinière (ICM), F-75013 Paris, France.

4. BCBL, Basque Center on Cognition, Brain and Language, Donostia, Spain.

Correspondence should be addressed to Ana B. Chica. Departamento de Psicología Experimental. Facultad de Psicología. Universidad de Granada. Campus de Cartuja S/N. CP 18071. Granada, Spain. Contact email: anachica@ugr.es.

Number of words for Abstract: 138; Introduction: 1799; Method: 2537; Results: 1451; Discussion: 938. Number of Figures: 2; Number of tables: 4.

The authors declare no conflict of interest. 


\begin{abstract}
Attention is considered as one of the pre-requisites of conscious perception. Phasic alerting and exogenous orienting improve conscious perception of near-threshold information, through segregated brain networks. Using a multimodal neuroimaging approach, combining data from functional MRI (fMRI) and diffusion-weighted imaging (DWI), we investigated the influence of white matter properties of the ventral branch of Superior Longitudinal Fasciculus (SLF III) in functional interactions between attentional systems and conscious perception. Results revealed that (1) reduced integrity of the left hemisphere SLF III was predictive of the neural interactions observed between exogenous orienting and conscious perception, and (2) increased integrity of the left hemisphere SLF III was predictive of the neural interactions observed between phasic alerting and conscious perception. Our results combining fMRI and DWI data demonstrate that structural properties of the white matter organization determine attentional modulations over conscious perception.
\end{abstract}

Keywords: exogenous attention, phasic alerting, consciousness, fMRI, Superior Longitudinal Fascicule (SLF). 
Perceptual consciousness refers to the ability to report, verbally or with an intended gesture, part of the information that is being processed in a given moment (Dehaene \& Changeux, 2011). Although it is highly possible that consciousness can be achieved without being able to report it, this condition is extremely difficult to study scientifically (although see Aru, Axmacher, et al., 2012; Koch, Massimini, Boly, \& Tononi, 2016; Sperling, 1960; Tsuchiya, Wilke, Frassle, \& Lamme, 2015). In the last decades, there has been an increased research interest on the neural correlates of consciousness (NCC) (Calabro, Cacciola, Bramanti, \& Milardi, 2015; Koch et al., 2016). The NCC is defined as the minimal set of neural events and mechanisms sufficient for a specific conscious percept (Koch et al., 2016). Some models claim that the NCC are primarily located in sensory regions (Pins \& Ffytche, 2003; Rees, Kreiman, \& Koch, 2002; Zeki, 2005), while others propose that the NCC are distributed in large-scale brain networks that allow the recurrent flow of information (Dehaene \& Changeux, 2011; Lamme, 2006; Rees et al., 2002). Moreover, theoretical and empirical claims have started to emerge, highlighting the importance of disentangling the NCC from its pre-requisites and consequences (Aru, Bachmann, Singer, \& Melloni, 2012). On the one hand, pre-requisites of consciousness are factors that modify consciousness thresholds, such as the minimal level of arousal necessary to process information, stimulus expectation, adaptation, working memory, or the allocation of attention. On the other hand, consequences of consciousness refer to the cascade of cognitive processes that follow conscious processing and that are directly enabled by it (Aru, Bachmann, et al., 2012; Seth, 2009). For example, when listening to a new word, we might want to keep it in working memory for further processing and encoding in long-term memory.

Here we aimed at investigating the pre-requisites of consciousness, rather than on the NCC itself or its consequences. According to several relevant models, attention is one of the pre-requisites of conscious perception (Dehaene \& Changeux, 2011; Dehaene, Changeux, Naccache, Sackur, \& Sergent, 2006; Mack \& Rock, 1998; Marois \& Ivanoff, 2005; Posner, 1994, 2012). Attentional processes can push perceptual information closer to, and sometimes above, the conscious threshold. However, attention is a heterogeneous construct, and recent research has shown that not all attentional subsystems enhance conscious perception behaviorally or overlap at the neural level with the NCC. In particular, while top-down or endogenous orienting (guided by goals or expectations) is clearly dissociable from consciousness (Koch \& 
Tsuchiya, 2007; Wyart, Dehaene, \& Tallon-Baudry, 2011; Wyart \& Tallon-Baudry, 2008), exogenous orienting and phasic alerting can increase perceptual sensitivity to detect near-threshold targets (Chica, Lasaponara, et al., 2011; Kusnir, Chica, Mitsumasu, \& Bartolomeo, 2011), and demonstrate neural interactions with consciousness (Chica, Bayle, Botta, Bartolomeo, \& Paz-Alonso, 2016; Chica, PazAlonso, Valero-Cabre, \& Bartolomeo, 2013). Exogenous orienting refers to the automatic allocation of attentional resources driven by salient external events at specific spatial regions (Chica, Bartolomeo, \& Lupiáñez, 2013). On the other hand, phasic alerting refers to the brief increase in arousal produced by the warning of an imperative stimulus (Petersen \& Posner, 2013; Sturm \& Willmes, 2001). Phasic alerting has been demonstrated to shorten response times (sometimes at the expense of accuracy) (Petersen \& Posner, 2013), but also to consistently improve the conscious perception of near-threshold information (Botta, Lupiáñez, \& Chica, 2014; Chica et al., 2016; Kusnir et al., 2011).

Both exogenous orienting and phasic alerting are often associated with the functioning of dorsal and ventral fronto-parietal networks. Orienting of attention is implemented by a dorsal, bilaterally distributed fronto-parietal network (Corbetta, Patel, \& Shulman, 2008; Corbetta \& Shulman, 2002). This network includes key regions, such as the frontal eye fields (FEF) and superior and inferior parietal lobes (SPL and IPL, respectively). The dorsal network might also be implicated in phasic alerting (Coull, Frith, Buchel, \& Nobre, 2000; Coull, Nobre, \& Frith, 2001; Sturm et al., 1999; Sturm et al., 2004; Sturm \& Willmes, 2001). On the other hand, the ventral fronto-parietal network is largely right lateralized and includes key regions such as the temporoparietal junction (TPJ) and inferior frontal gyrus (IFG). Activity in the ventral network has been related to exogenous orienting in healthy participants (Chica, Bartolomeo, \& Valero-Cabré, 2011; Shulman et al., 2009; Shulman, Astafiev, McAvoy, d'Avossa, \& Corbetta, 2007) and in neglect patients (Bartolomeo, 2007; Bartolomeo, Thiebaut de Schotten, \& Chica, 2013; Bartolomeo, Thiebaut de Schotten, \& Doricchi, 2007; Corbetta et al., 2008), while its role in phasic alerting is less known (but see Kim, 2014).

In two previous functional MRI (fMRI) studies, we investigated the interactions between exogenous orienting and phasic alerting systems with conscious perception. In a first study (Chica, Paz-Alonso, et al., 2013), we used a spatially informative peripheral 
cue, presented either at the target side or at the opposite location, to explore the neural networks underlying the interactions between exogenous orienting and conscious perception. Behaviorally, target contrast to perceive $\sim 50 \%$ of the target-present trials resulted to be lower for valid as compared to invalid locations. The left FEF and right inferior parietal sulcus (IPS) were two of the regions demonstrating interactions between exogenous orienting ${ }^{1}$ and conscious perception. These regions were more activated for seen trials than for unseen trials, and this effect was larger when participants were paying attention to the location indicated by the cue (valid trials) than when they attended the opposite location (invalid trials). Functional connectivity analyses also showed that these regions (together with some other fronto-parietal regions) were more strongly coactivated for seen than for unseen trials in the valid condition. However, the effect reversed in the invalid condition, where these regions were more strongly coupled to unseen than to seen trials. Thus, according to these results, fronto-parietal interactions are not only relevant for the conscious perception of near-threshold targets, but also for attentional orienting, before target presentation, facilitating access to consciousness for spatially attended targets.

In a second study (Chica et al., 2016), we manipulated the presence of an auditory tone to examine the neural networks underlying the interactions between phasic alerting and conscious perception (endogenous orienting was also manipulated in this experiment, although it only demonstrated a weak interaction with conscious perception in the thalamus). Behaviorally, Gabor contrast to perceive $\sim 50 \%$ of the targets was lower for the tone as compared to the no-tone condition (Botta et al., 2014; Kusnir et al., 2011). The caudate nucleus, anterior cingulate cortex (ACC), supplementary motor area (SMA), and FEF were the key regions demonstrating interactions between phasic alerting and consciousness. These regions were more engaged for seen as compared to unseen trials, especially when no tone was presented. Functional connectivity analyses also showed that the ACC and SMA were more strongly coupled for seen as compared to unseen trials, especially in the no-tone

\footnotetext{
${ }^{1}$ Note that although cues were spatially informative, they consisted of peripheral and salient stimuli, which are known to exogenously capture attention to that location. I.e. peripheral informative cues produce both an initial exogenous attentional capture, and an endogenous maintenance of attention at the indicated location (Chica, et al., 2013a). Therefore, although we refer to Chica, et al.'s (2013b) study as the "Exogenous Orienting" study, we acknowledge there is an endogenous maintenance component in the orienting of attention.
} 
condition. These results suggested that, in the absence of external alerting tones, stimuli were more likely to be detected if alerting mechanisms were endogenously activated during the cue period.

Anatomically, dorsal and ventral fronto-parietal networks communicate through large white-matter fiber bundles that have been well described in humans using diffusion-weighted imaging (DWI) tractography. Fronto-parietal cortical regions are organized along three longitudinal fiber tracts separated into a dorsal superior longitudinal fasciculus (SLF I), a middle branch (SLF II), and a ventral branch (SLF III) (Thiebaut de Schotten et al., 2011). Cortical projections of the SLF I are usually symmetrically distributed between the left and right hemisphere, overlapping with the dorsal network. The SLF III is more right lateralized, overlapping with the ventral network. The SLF II is also right lateralized, and overlaps with the parietal component of the ventral network and the prefrontal component of the dorsal network, probably affording direct communication between ventral and dorsal attentional networks (Thiebaut de Schotten et al., 2011).

Exogenous orienting has been reliably associated with the white matter microstructure of the SLF I and III, both in normal observers (Carretie, Rios, Perianez, Kessel, \& Alvarez-Linera, 2012; Thiebaut de Schotten et al., 2011) and in braindamaged patients with signs of left neglect (Bourgeois et al., 2015; Bourgeois, Chica, Migliaccio, Thiebaut de Schotten, \& Bartolomeo, 2012; Doricchi, Thiebaut de Schotten, Tomaiuolo, \& Bartolomeo, 2008; Thiebaut de Schotten et al., 2014; Urbanski et al., 2008). Damage to the more ventral branch of SLF (i.e., SLF III) has been proposed to play a key role in left neglect, which is consistent with the idea that the ventral network plays a crucial role in exogenous spatial attention, especially damaged in neglect (Ciaraffa, Castelli, Parati, Bartolomeo, \& Bizzi, 2013; Urbanski et al., 2011). In fact, the integrity of the dorsal network in neglect might explain patients' spared abilities to endogenously orient attention in space (Bartolomeo, Siéroff, Decaix, \& Chokron, 2001). The alerting system, on the other hand, has been consistently associated with the whitematter microstructure of the internal capsule (Ge et al., 2013; Niogi, Mukherjee, Ghajar, \& McCandliss, 2010), and there is some evidence in children on the role of the right SLF in sustained attention (also known as vigilance) (Klarborg et al., 2013). However, there is currently no available evidence relating phasic alerting with SLF structure. 
The present study was aimed at investigating in the healthy brain the influence of the ventral branch of the SLF (i.e., SLF III) white-matter microstructure in the bloodoxygen-level dependent (BOLD) interactions observed in the two previous fMRI studies (Chica et al., 2016; Chica, Paz-Alonso, et al., 2013) on the conscious perception of near-threshold targets. We decided to focus on SLF III for the following reasons: (1) studies in neglect patients (Bartolomeo \& Thiebaut de Schotten, 2016; Bartolomeo et al., 2007; Ciaraffa et al., 2013; Urbanski et al., 2011) and studies using a transcranial magnetic stimulation approach (Chica, Bartolomeo, et al., 2011) have demonstrated the critical role of the ventral network in exogenous orienting. However, the role of SLF III in conscious perception is less known. (2) The ventral network has also been related to the alerting system (Corbetta \& Shulman, 2002; Downar, Crawley, Mikulis, \& Davis, 2002; Shinoura et al., 2009; Uddin, 2015), although the role of SLF III in phasic alerting remains largely unexplored.

We hypothesized that right SLF III microstructure might predict the functional interactions previously observed between exogenous orienting and conscious perception in fronto-parietal regions (Bartolomeo et al., 2013; Chica, Bartolomeo, et al., 2013; Chica, Bartolomeo, et al., 2011; Corbetta \& Shulman, 2002). The engagement of the ventral network has also been associated to alerting (Corbetta \& Shulman, 2002; Downar et al., 2002; Kim, 2014; Shinoura et al., 2009; Uddin, 2015), and although the ventral network is usually right-lateralized, fMRI evidence also suggests a role for lefthemisphere fronto-parietal activations in phasic alerting (Coull et al., 2001), or in attentional selection (Sturm and Willmes, 2001). We therefore hypothesized that left SLF III structure might predict a more effective use of phasic alerting signals in attentional selection.

\section{Methods}

\section{Participants}

DWI data from one participant in the phasic alerting experiment and two participants in the exogenous orienting experiment were lost or presented artifacts, which precluded their analyses. Data from eighteen voluntaries were analyzed in the phasic alerting experiment (mean age 26 years, standard deviation $[\mathrm{SD}]=5 ; 10$ females). In the exogenous orienting experiment, data from sixteen voluntaries were analyzed (mean age 25 years, $\mathrm{SD}=5 ; 11$ females). All participants were right-handed 
and lived in Paris, France. They had no neurological or psychiatric conditions and followed all the safety requirements to undergo MRI scanning. All participants were naive to the purpose of the experiment, reported to have normal or corrected-to-normal vision, and received a monetary compensation for their participation. They gave signed informed consent to participate in the study. The study was reviewed by the INSERM ethical committee and received the approval of an Institutional Review Board (CPP Ile de France 1, Paris, France). The study was carried out in accordance with the approved guidelines.

\section{Stimuli, and procedure}

Figure 1 describes the paradigms used in both studies. In the exogenous orienting experiment (Figure 1A), a peripheral cue, consisting of a square surrounding one of the peripheral markers, was used to attract spatial attention exogenously. This cue was predictive about the spatial location of the target on $67 \%$ of the target-present trials. Participants were informed about the predictive value of the cue, although they were not told the exact amount of trials in which the cue predicted the target's location. They were encouraged to take this information into account in order to respond more accurately. The target consisted of a grating, which contrast was titrated so that it would be consciously seen in only $50 \%$ of the trials (see titration procedure below). No target was presented on $14 \%$ of the trials.

In the phasic alerting experiment (Figure 1B), endogenous attention was manipulated before the auditory tone was presented using a central symbolic cue. Cue color predicted the spatial location of the target on $70 \%$ of the target-present trials. Participants were informed about the predictive value of the cue. Although they were not told the exact amount of trials in which the cue predicted the target's location, they were encouraged to take this information into account in order to respond more accurately. The alerting cue was presented on $50 \%$ of the trials, and consisted of white noise $(22.050 \mathrm{~Hz}, 74 \mathrm{~dB})$ presented through headphones. The target consisted of a Gabor, which contrast was titrated so that it would be consciously seen in only $50 \%$ of the trials (see titration procedure below). No target was presented on $13 \%$ of the trials. 
Please insert Figure 1 about here

In both experiments, participants were asked to provide two responses to each target consecutively, by making key presses on a 2-horizontally-aligned-button fiberoptic box. First, they were required to discriminate the orientation of the target (objective task) by pressing, with their right hand, a left situated key if the target was oriented to the left, and a right situated key if the target was oriented to the right. Participants were encouraged to respond to every trial as fast and accurately as possible. Even if they did not see the stimulus, they were encouraged to guess the correct response.

Second, participants had to report if they consciously detected the appearance of the target (subjective task) as accurately as possible. This time, we encouraged participants to take their time to respond correctly and to report the presence of the target when they were confident about it. In the phasic alerting experiment, we presented participants with two arrow-like stimuli, one below and the other one above the fixation point $(>>>$ or $<<<$ ). The vertical arrangement of the arrow-like stimuli ensured that participants could not prepare a lateralized response in advance, associated with the location of the target. We provided participants with 3 vertically aligned keys (to-be-pressed using the left hand). The upper key always corresponded to the arrow presented above the fixation point; the middle key was associated with the arrow presented below the fixation point; and, the lower key was used to indicate that the target was not seen. In target-absent trials, participants were also required to give the objective response, and then report whether they saw the target or not. In the exogenous orienting experiment participants reported the conscious perception of the target when they saw the question: "Did you see the stimulus?" (subjective task) and the French words for "yes" and "no" situated below the question. As in the phasic alerting experiment, for target-absent trials, participants were required to give the objective response and to report that no target was seen in the subjective response.

In order to present the target stimuli at the threshold of conscious perception, target contrast in both experiments was adjusted at the beginning of the fMRI session, so that the percentage of consciously perceived targets was $\sim 50 \%$ for each experimental condition. This titration procedure was done based on individuals' performance on a 
titration block that was run before the experimental task. Titration was carried out independently for each experimental condition. All participants started with a high contrast stimulus, which was well above the threshold of conscious perception. After each titration block, target contrast was automatically adjusted using a "one-up-onedown" procedure, until participants perceived $\sim 50 \%$ of targets for each condition in at least two consecutive blocks of trials. If the percentage of correct detection rates was above $55 \%$ of the trials, targets at the immediately following lower contrast level were used for the next block. Inversely, if the percentage of correct detection rates was below $45 \%$ of the trials, targets at the immediately following higher contrast level were used for the next block. The experimental session started when participants felt comfortable with the task, and performance converged at a target contrast yielding $\sim 50 \%$ seen targets for each condition. This titration procedure continued during the whole experiment to prevent factors such as practice or fatigue from influencing conscious perception.

The exogenous orienting experiment consisted of a total of 280 trials presented in 5 functional scans. Each of these 5 functional scans lasted $7 \mathrm{~min}$. Valid trials were twice more likely than invalid trials. The phasic alerting experiment consisted of two sessions with 5 functional scans each. Each functional scan lasted $12 \mathrm{~min}$. They performed the task twice in two different fMRI sessions. For both sessions, participants encountered a total of 920 trials (120 of them were target-absent trials). Valid trials accounted for $70 \%$ of the target-present trials. In both experiments, each trial type was presented in a pseudorandomized order during scanning. The jitter fixation and the order of trial types within each scan were determined with an optimal sequencing program designed to maximize the efficiency of the estimation of the blood oxygen level-dependent (BOLD) response (Optseq II; Dale, 1999). The jitter fixation periods were interleaved with the experimental trials as determined by the optimization program.

\section{MRI data acquisition}

The functional and structural T1-weighted sequences used for the phasic alerting and exogenous orienting studies were practically identical, and are reported in previous publications (Chica et al., 2016; Chica, Paz-Alonso, et al., 2013). A fully optimized acquisition sequence for the tractography of DWI was employed, which provided 
isotropic $(2 \times 2 \times 2 \mathrm{~mm})$ resolution and coverage of the whole head with a posterioranterior phase of acquisition. A total of 70 near-axial slices were acquired on a Siemens 3 Tesla TRIO TIM system equipped with a 32-channel head coil. We used an echo time (TE) of $88 \mathrm{msec}$ and a repetition time (TR) of $8400 \mathrm{msec}$. At each slice location, 6 images were acquired with no diffusion gradient applied. Additionally, 60 diffusionweighted images were acquired. The diffusion weighting was equal to a b-value of 1500 sec $\mathrm{mm}^{2}$. At each slice, diffusion-weighted data were simultaneously registered and corrected for subject motion and geometrical distortion adjusting the gradient accordingly (ExploreDTI http://www.exploredti.com; Leemans \& Jones, 2009).

\section{MRI data analyses}

Spherical Deconvolution Tractography reconstruction. Damped Richardson Lucy Spherical Deconvolution (Dell'Acqua et al., 2010) was computed to estimate multiple orientations in voxels containing different populations of crossing fibers. Algorithm parameters were chosen, as previously described (Dell'Acqua, Simmons, Williams, \& Catani, 2013). A fixed-fiber response corresponding to a shape factor of a $=2 \times 10-3 \mathrm{~mm}^{2} / \mathrm{s}$ was chosen (Dell'Acqua et al., 2013).

Whole-brain tractography was performed selecting every brain voxel with at least one fiber orientation as a seed voxel. From these voxels, and for each fiber orientation, streamlines were propagated using Euler integration with a step size of 1 mm (Dell'Acqua et al., 2013). When entering a region with crossing white matter bundles, the algorithm followed the orientation vector of least curvature. Streamlines were halted when a voxel without fiber orientation was reached or when the curvature between two steps exceeded a threshold of $45^{\circ}$. Spherical deconvolution, fiber orientation vector estimations and tractography were performed using Startrack (http://www.natbrainlab.co.uk).

Tractography dissections. In order to facilitate the tractography dissection, regions of interest (ROI) were defined on the CS-MNI template calculated above, based on the guidelines provided in previous reports (Rojkova et al., 2016; Thiebaut de Schotten et al., 2011). For each participant, the CS Map was registered to the CSMNI152 template using ANTs.

Tract-specific measures of tract microstructural organization (i.e. mean Hindrance Modulated Orientational Anisotropy or HMOA for the whole tract) 
(Dell'Acqua et al., 2013) were extracted from each dissected tract. HMOA provides information about the microstructural diffusion properties of distinct fiber orientations and therefore specific to the orientation of the reconstructed tracts and more accurate than classical fractional anisotropy measures, which decreases when fibers cross due to local partial volume effect.

fMRI data preprocessing. Standard preprocessing routines were conducted in SPM8 (Welcome Department of Cognitive Neurology, London). Images were corrected for differences in timing of slice acquisition and were realigned to the first volume by means of rigid-body transformation. Then, functional images were spatially smoothed using a 4-mm full width at half-maximum (FWHM) isotropic Gaussian kernel. Next, motion parameters obtained from realignment were used to inform a volume repair procedure (ArtRepair; Stanford Psychiatric Neuroimaging Laboratory) that identified bad volumes on the basis of within-scan movement and signal fluctuations, and then corrected bad signal values via interpolation. A volume-by-volume correction with a 1.5 $\mathrm{mm}$ threshold was applied, which did not remove more than $15 \%$ of the volumes in any participant of the final study sample. After volume repair, structural and functional volumes were coregistered and spatially normalized to $\mathrm{T} 1$ and echo-planar imaging templates, respectively. The normalization algorithm used a 12-parameter affine transformation together with a non-linear transformation involving cosine basis functions. During normalization, the volumes were sampled to 3-mm cubic voxels. Templates were based on the MNI305 stereotaxic space. Then, functional volumes were spatially smoothed with a 7-mm FWHM isotropic Gaussian kernel. Finally, time series were temporally filtered to eliminate contamination from slow frequency drift (highpass filter with cut-off period: $128 \mathrm{sec}$ ).

ROI analysis. Statistical analyses were performed on individual participants' data using the general linear model (GLM). fMRI time series data were modeled by a series of events convolved with a canonical hemodynamic response function (HRF). ROI analyses were performed with the MARSBAR toolbox. ROIs consisted on 5-mm radius spheres centered at local maxima found in previous studies. Two ROIs were selected for each study. We decided not to extract these ROIs directly from our previous studies to avoid circularity by violating the assumption of random sampling. For the exogenous orienting study, the ROIs corresponded to the left FEF, the region demonstrating the larger interaction between exogenous orienting and consciousness, 
and the right IPS, another region of the fronto-parietal network demonstrating interactions between exogenous orienting and consciousness. Both are known to be key regions for attentional orienting. For the phasic alerting study, the ROIs corresponded to the left ACC, the region demonstrating the larger interaction between phasic alerting and consciousness, and the left FEF, another region of the fronto-parietal network demonstrating interactions between phasic alerting and consciousness. Both are known to be key regions for alerting.

Left FEF and right IPS were selected from a seminal study highlighting the contributions of the dorsal and ventral network to different types of spatial orienting (Kincade, Abrams, Astafiev, Shulman, \& Corbetta, 2005; left FEF: $x=-24, y=-3, z=57$; right IPS: $x=15, y=-67, z=53$ ). Left ACC was selected from an fMRI study exploring the neural correlates of phasic alerting (Yanaka, Saito, Uchiyama, \& Sadato, 2010; left ACC: $x=-6, y=10, z=44$ ), while the right caudate was selected from an fMRI study exploring the role of serotonie in sustained attention (Wingen, Kuypers, van de Ven, Formisano, \& Ramaekers, 2008; MNI coordinates $\mathrm{x}=10, \mathrm{y}=7, \mathrm{z}=7$ ).

Classical Lineal Regression and Bayesian Regression analyses. In order to examine the role of the SLF III in the functional interactions observed between attention (exogenous orienting and phasic alerting) and consciousness, we performed both a classical lineal regression (using the "backward" method) and Bayesian regression analyses. In Bayesian statistics, analyses are not biased against the null hypothesis, and we can establish evidence for the absence of an effect only on the observed data. Therefore, with the observed data, we can conclude if the alternative hypothesis is more probable than the null hypothesis or vice-versa.

The exogenous orienting and phasic alerting studies had different independent variables, and neural interactions were observed in different brain regions. We used a similar approach in both studies, although adapting the analyses to the design of each study and critical regions observed in our previous fMRI results. In both classical and Bayesian regressions, we used as dependent variable an index based on the parameter estimates of the functional interaction between attention and consciousness observed in the brain region showing the most robust interaction. As predictors we used an index based on the parameter estimates of the attention and consciousness interaction observed in another key brain region, an index of the behavioral interaction, and the 
HMOA of the SLF III in the left and right hemisphere. All data were normalized by Z score transformations.

In the exogenous orienting study, we calculated an index of the functional interaction in the left FEF and right IPS.

$$
\text { PE (VS - VU) + PE (IU - IS) }
$$

PE: parameter estimate; VS: valid seen; VU: valid unseen; IS: invalid seen; IU: invalid unseen.

We calculated a similar index of the behavioral interaction:

$$
\% \text { seen targets }(V S-V U)+\% \text { seen targets (IU - IS) }
$$

Therefore, in the exogenous orienting study we tried to predict the functional interaction observed in the left FEF, using as predictors the functional interaction of the right IPS, the behavioral interaction, and the HMOA of the SLF III in the left and right hemisphere.

For the phasic alerting study, we calculated an index of the functional interaction in the left ACC and right caudate.

$$
\text { PE }(\text { ToS }- \text { ToU) }+ \text { PE }(\text { NoToS }- \text { NoToU) }
$$

PE: parameter estimate; ToS: Tone-Seen; ToU: Tone-Unseen; NoToS: No Tone-Seen; NoToU: No Tone-Unseen.

We calculated a similar index of the behavioral interaction:

$$
\% \text { seen targets }(\mathrm{ToS}-\mathrm{ToU})+\% \text { seen targets (NoToS }- \text { NoToU) }
$$

Therefore, in the phasic alerting study we tried to predict the functional interaction observed in the left $\mathrm{ACC}$, using as predictors the functional interaction of the right caudate, the behavioral interaction, and the HMOA of the SLF III in the left and right hemisphere. 


\section{Results}

As reported in the previous publication (Chica et al., 2013), behavioral results demonstrated that spatial orienting improved target conscious detection rates. Target contrast fulfilling the fixed threshold of 50\% correct conscious detection (subjective task) proved lower for valid than for invalid trials, and this difference was larger for unseen targets than for seen targets (Fig. 2B, Chica et al., 2013). ROI analyses demonstrated that the left FEF and the right IPL showed a significant interaction between Validity and Awareness, being more strongly engaged for trials involving targets reported as seen than for unseen targets, but this time only when the cue correctly oriented attention toward the target location (Fig. 3, Chica et al., 2013).

In the phasic alerting study (Chica et al., 2016), Gabor contrast to perceive $\sim 50 \%$ of the targets resulted to be lower for tone present as compared to tone absent conditions (Fig. 2, Chica et al., 2016). ROI analyses revealed a group of regions showing a statistically significant Alerting State x Awareness interaction, including bilateral ACC, caudate, FEF, and SMA (see Table 2 and Fig. 3B, Chica et al., 2016). BOLD activation was larger for seen as compared to unseen reports in all the above-mentioned regions. The effect was larger in no tone trials as compared to tone present trials.

The tractography results demonstrated that SLF III was right lateralized in our sample of participants (mean HMOA right hemisphere $=0.097$, mean HMOA left hemisphere $=0.090$; $t$-student, $p=0.004$; see Figure 2), confirming results from previous studies (Thiebaut de Schotten et al., 2011). Laterality of the fascicule (HMOA right hemisphere minus HMOA left hemisphere) was correlated with the HMOA from both the left and right hemispheres to explore whether the lateralization of SLF III was related to reduced integrity of the left hemisphere or to increased integrity of the right hemisphere. Pearson correlations demonstrated that the laterality of the fascicule was significantly (and negatively) correlated with the integrity of SLF III in the left hemisphere $(r=-0.731, p<0.001)$ but not with the integrity of SLF III in the right hemisphere $(\mathrm{r}=0.103, \mathrm{p}=.564)$.

\section{Exogenous Orienting study}

Results of the classical general lineal regression analysis are summarized in Table 1. We observed significant contributions of three variables to the left FEF functional interaction used as the dependent variable: HMOA of the left SLF III, the 
behavioral interaction, and the functional interaction in the right IPS (see Table 1). For the left SLF III, the beta value was negative, indicating that a reduced integrity of SLF III in the left hemisphere predicts the functional interaction between orienting and consciousness in the left FEF (see Figure 2). As expected, the larger was the functional interaction in the right IPS, the larger was the functional interaction in the left FEF.

Bayesian statistics confirmed this result. The largest Bayesian factor was associated with the combination of the HMOA on the left SLF III, the behavioral interaction, and the functional interaction in the right IPS $\left(\mathrm{BF}_{\mathrm{M}}=3.947^{2}\right)$. The next model with the largest Bayesian factor was the model taking into account the HMOA on the left SLF III, HMOA on the right SLF III, the behavioral interaction, and the functional interaction in the right IPS $\left(\mathrm{BF}_{\mathrm{M}}=3.740\right.$; all other values $\left.<1.805\right)$. However, the model taking into account the integrity of the right SLF III did not add much predictive value, neither to the classical regression nor to the Bayesian regression analyses. Therefore, these data suggests that a reduced integrity of the left SLF III was predictive of the functional interaction observed in the left FEF.

Therefore, an asymmetry of SLF III, with reduced integrity of the left hemisphere SLF III as compared to the right hemisphere SLF III, was predictive of the functional interaction we observed in the left FEF. These results confirm the substantial right lateralization (reduced integrity of the left branch) of the fronto-parietal networks for the use of orienting signals, adding evidence from white matter micro-structure to the previous observations in brain damaged patients and fMRI studies (Bourgeois, Chica, Valero-Cabre, \& Bartolomeo, 2013a, 2013b; Corbetta et al., 2008; Corbetta \& Shulman, 2002).

Please insert Table 1 and Figure 2 about here

\footnotetext{
${ }^{2}$ In Bayesian statistics a Bayesian Factor $=1$ indicates no evidence in favor of either the null or the alternative hypothesis. Bayesian Factors $>3$ indicate moderate evidence in favor of the alternative hypothesis, while Bayesian Factors $<-3$ indicate moderate evidence in favor of the null hypothesis. Bayesian Factors values between -3 and 3 indicate anecdotal evidence.
} 


\section{Control analyses}

When the same analysis was performed using the integrity of the SLF I rather than the SLF III as a predictor, the classical lineal regression model demonstrated that this factor was not predictive at all of the functional interaction observed in the left FEF (see Table 2). The only factor that predicted the left FEF interaction was the functional interaction in the right IPS. A Bayesian analysis confirm that the largest Bayesian factor was associated with the functional interaction observed in the right IPS $\left(\mathrm{BF}_{\mathrm{M}}=2.599\right.$; all other $\mathrm{BF}_{\mathrm{M}}<2.285$ for the combination of the functional interaction observed in the right IPS and the behavioral effect).

Finally, we repeated the classical lineal regression and Bayesian regression analyses but using the behavioral index as the dependent variable. This analysis was meant to understand if differences in SLF III could also predict behavioral differences in conscious perception or attentional abilities. The functional interaction of the left FEF and right IPS, and the HMOA of the SLF III in the left and right hemisphere were used as predictors in the exogenous orienting study. None of the models resulted significant in the analyses (all ps> .101 and all $\mathrm{BF}_{\mathrm{M}}>2.144$ ). Therefore, integrity of the SLF III was predictive of the functional interactions observed in the left FEF but not of the behavioral results of the present set of data.

\section{Phasic alerting study}

Results of the classical general lineal regression analysis are summarized in Table 3. We observed significant contributions of two variables to the left ACC functional interaction used as the dependent variable: HMOA of the left SLF III and the functional interaction in the right caudate (see Table 3). For the left SLF III, the beta value was positive, indicating that a larger integrity of SLF III in the left hemisphere predicts the functional interaction between phasic alerting and consciousness in the left ACC (see Figure 2). As expected from the fMRI results, the larger the functional interaction in the right caudate, the larger the functional interaction in the left ACC.

Please insert Table 3 and Figure 2 about here

Bayesian statistics confirmed this result. The largest Bayesian factor was associated with the combination of the left SFL III and the functional interaction in the 
right caudate nucleus $\left(\mathrm{BF}_{\mathrm{M}}=5.398\right.$, all other values $\left.<2.001\right)$. This result indicates that the integrity of the left SLF III and the functional interaction in the right caudate can predict the functional interaction in the left ACC, and this model is 5.398 more likely than the null hypothesis. This is considered as moderate evidence in favor of the alternative hypothesis.

These results demonstrate the importance of the left ventral network for the use of alerting signals. Despite the overall right lateralization of SLF III, in this study, the integrity of the left SLF III was associated with increased neural interactions between alerting and conscious perception, which is consistent with the left-lateralization of the phasic alerting network reported in previous fMRI studies (Coull et al., 2001).

\section{Control analyses}

When the same analysis was performed using the integrity of the SLF I rather than the SLF III as a predictor, the classical lineal regression model demonstrated that this factor was not predictive at all of the functional interaction observed in the left ACC (see Table 4). The only factor that predicted the left ACC interaction was the functional interaction in the right caudate. A Bayesian analysis confirmed that the largest Bayesian factor was associated with the functional interaction observed in the right caudate $\left(\mathrm{BF}_{\mathrm{M}}=3.314\right.$; all other $\mathrm{BF}_{\mathrm{M}}<2.319$ for the combination of the functional interaction observed in the right caudate and the integrity of the left SLF I). Note that the latter effect with $\mathrm{BF}_{\mathrm{M}}<3$ indicates only anecdotal evidence.

Finally, we repeated the classical lineal regression and Bayesian regression analyses but using the behavioral index as the dependent variable to understand if the differences in SLF III could also predict behavioral differences in conscious perception or attentional abilities. The functional interaction of the left ACC and right caudate, and the HMOA of the SLF III in the left and right hemisphere were used as predictors in the phasic alerting study. None of the models resulted significant in the analyses (all ps> .553). The Bayesian lineal regression analysis demonstrated moderate evidence in favor of the null hypothesis $\left(\mathrm{BF}_{\mathrm{M}}=3.124\right)$. Therefore, integrity of the SLF III was predictive of the functional interactions observed in the left ACC but not of the behavioral results of the present set of data. 


\section{Discussion}

The aim of the present study was to investigate the influence of white matter microstructure of the ventral branch of SLF (i.e. SLF III) on the interactions between attention and consciousness that we have previously observed in two fMRI studies (Chica et al., 2016; Chica, Paz-Alonso, et al., 2013). Our previous results showed that segregated cortical networks support the interactions between attentional systems and conscious perception. The main findings of the present study concern the predictive values of the integrity of SLF III in the left hemisphere for the effects of exogenous attention and phasic alerting on conscious perception. While a reduced integrity of SLF III in the left hemisphere was related to increased functional interactions between attention and consciousness, an increased integrity of this same fasciculus in the left hemisphere was related to increased functional interactions between phasic alerting and consciousness. These results are in agreement with previous studies, which have reported similar lateralization patterns using fMRI (for exogenous orienting: Bartolomeo et al., 2013; Bourgeois et al., 2013b; Corbetta et al., 2008) (for alerting: Clemens et al., 2011; Coull et al., 2000; Coull et al., 2001; Sturm et al., 2004; Thiel \& Fink, 2007), and DWI (for orienting: Thiebaut de Schotten et al., 2011; Thiebaut de Schotten et al., 2005). However, to the best of our knowledge there was no available evidence concerning the influence of SLF structure on phasic alerting, and concerning the interactions between attention and consciousness relating relevant fMRI and DWI indexes.

A current controversy exists about the neural basis of the exogenous orienting of spatial attention. Using fMRI, Corbetta and colleagues (2008) have proposed a very influential model, according to which, orienting of attention (whether endogenous or exogenous), is implemented in the dorsal fronto-parietal networks. The ventral frontoparietal network is typically associated with attentional re-orienting to task-relevant events. Nonetheless, as previously noticed (Chica, Bartolomeo, et al., 2011), the insufficient temporal resolution of fMRI prevents the capture of fast and brief neural events, such as exogenously driven attentional orienting, which peaks $100 \mathrm{~ms}$ after cue onset (Müller \& Rabbitt, 1989). Using TMS during the orienting of attention, Chica et al. (2011) demonstrated that causal interference of the right TPJ (a key region of the ventral network) altered the orienting of exogenous but not endogenous attention. Consistent with this observation, damage to the right TPJ and its connections to the 
frontal cortex through the SLF largely impairs exogenous orienting (and consequently conscious perception) in neglect patients (Bartolomeo et al., 2013; Bartolomeo et al., 2007; Bourgeois et al., 2012; Thiebaut de Schotten et al., 2014). The results of the present work add to these observations, demonstrating that a reduced integrity of the left SLF III was associated with a larger interaction between exogenous orienting and consciousness in a key region of the fronto-parietal network: the left FEF. Previous evidence gathered by using TMS (Chica, Valero-Cabré, Paz-Alonso, \& Bartolomeo, 2014) converged in demonstrating a crucial role of the left FEF in the interactions between attention and conscious perception. Given the importance right lateralization of the ventral network in exogenous attentional orienting, we hypothesized that right SLF III microstructure might predict the functional interactions between exogenous orienting and conscious perception in fronto-parietal regions (Bartolomeo et al., 2013; Chica, Bartolomeo, et al., 2013; Chica, Bartolomeo, et al., 2011; Corbetta \& Shulman, 2002). However, reduced integrity of the left SLF III (rather than increased integrity of the right branch) was associated to increased functional interactions between exogenous orienting attention and consciousness in the left FEF. More research is needed to confirm this new finding.

Interestingly, a right lateralized network does not seem to be equally beneficial for all attentional processes. Increased integrity of the left SLF III predicted larger functional interactions for the use of phasic alerting signals. These results are consistent with previous literature demonstrating a left lateralization of the fronto-parietal network associated with phasic alerting in fMRI studies (Clemens et al., 2011; Coull et al., 2000; Coull et al., 2001; Sturm et al., 2004; Thiel \& Fink, 2007). These new results are consistent to some extent with the alerting deficits observed after right hemisphere damage leading to neglect (Manly, Dobler, Dodds, \& George, 2005). Neglect patients present severe problems in sustaining attention over time (Robertson, Tegnér, Tham, Lo, \& Nimmo-Smith, 1995; Thimm, Fink, Kust, Karbe, \& Sturm, 2006), which have been associated with the damage to the SLF (Klarborg et al., 2013). Perhaps drawing on these left hemisphere resources (Bartolomeo \& Thiebaut de Schotten, 2016), phasic alerting can improve neglect deficits (Bartolomeo \& Thiebaut de Schotten, 2016; Chica, Thiebaut de Schotten, et al., 2011; Robertson, Mattingley, Rorden, \& Driver, 1998).

Our results combining fMRI and DWI data add important evidence to the existing literature demonstrating that structural properties of the white matter 
organization might determine attention and consciousness interactions. In the present research, two independent groups of participants took part in the exogenous orienting and phasic alerting studies. Future research should aim at comparing different tasks within the same participants, to directly test the hypothesis that white matter properties of the brain can predict different behavioral outcomes in individual participants (Bartolomeo, Seidel Malkinson, \& de Vito, 2017).

A further research question prompted by the present results concern the ways in which white matter microstructure in the damaged and healthy hemisphere might determine behavioral deficits after brain damage and, therefore, should be taken into account for rehabilitation purposes. For example, white matter microstructure may help predicting the evolution of cognitive and neurological deficits after brain injury (Bartolomeo \& Thiebaut de Schotten, 2016; Forkel et al., 2014; Lunven et al., 2015) and, thus, suggest the most appropriate strategies of rehabilitation. 


\section{Acknowledgements}

ABC was supported by a Ramón y Cajal fellowship (RYC-2011-09320) and research project PSI2014-58681-P from the Spanish Ministry of Economy and Competitiveness (MINECO). PMP-A was supported by a Ramón y Cajal fellowship (RYC-2014-15440), and grants PSI2015-65696 and SEV-2015-049 from the MINECO. MTdS received funding from the 'Agence Nationale de la Recherche' [grant number ANR-13-JSV40001-01] and "Investissements d'avenir" ANR-10-IAIHU-06. 


\section{References}

Aru, J., Axmacher, N., Do Lam, A. T., Fell, J., Elger, C. E., Singer, W., \& Melloni, L. (2012). Local category-specific gamma band responses in the visual cortex do not reflect conscious perception. Journal of Neuroscience, 32(43), 14909-14914. doi: 10.1523/JNEUROSCI.2051-12.2012

Aru, J., Bachmann, T., Singer, W., \& Melloni, L. (2012). Distilling the neural correlates of consciousness. Neuroscience and Biobehavioral Reviews, 36(2), 737-746. doi: S0149-7634(11)00210-7 [pii] 10.1016/j.neubiorev.2011.12.003

Bartolomeo, P. (2007). Visual neglect. Current Opinion in Neurology, 20(4), 381-386.

Bartolomeo, P., Seidel Malkinson, T., \& de Vito, S. (2017). Botallo's error, or the quandaries of the universality assumption. Cortex, 86, 176-185. doi: doi:10.1016/j.cortex.2016.09.026

Bartolomeo, P., Siéroff, E., Decaix, C., \& Chokron, S. (2001). Modulating the attentional bias in unilateral neglect: The effects of the strategic set. Experimental Brain Research, 137(3-4), 432-444.

Bartolomeo, P., \& Thiebaut de Schotten, M. (2016). Let thy left brain know what thy right brain doeth: Inter-hemispheric compensation of functional deficits after brain damage. Neuropsychologia, 93, 407-412. doi: 10.1016/j.neuropsychologia.2016.06.016

Bartolomeo, P., Thiebaut de Schotten, M., \& Chica, A. B. (2013). Brain networks of visuospatial attention and their disruption in visual neglect. Frontiers in Human Neuroscience, 6, 110. doi: 10.3389/fnhum.2012.00110

Bartolomeo, P., Thiebaut de Schotten, M., \& Doricchi, F. (2007). Left unilateral neglect as a disconnection syndrome. Cerebral Cortex, 17(11), 2479-2490.

Botta, F., Lupiáñez, J., \& Chica, A. B. (2014). When endogenous spatial attention improves conscious perception: Effects of alerting and bottom-up activation. Conscioussness and Cognition, 23, 63-73. doi: S1053-8100(13)00162-1 [pii] 10.1016/j.concog.2013.12.003

Bourgeois, A., Chica, A. B., Migliaccio, R., Bayle, D. J., Duret, C., Pradat-Diehl, P., . . Bartolomeo, P. (2015). Inappropriate rightward saccades after right hemisphere damage: Oculomotor analysis and anatomical correlates. Neuropsychologia, 73, 1-11. doi: S0028-3932(15)00159-1 [pii]

10.1016/j.neuropsychologia.2015.04.013

Bourgeois, A., Chica, A. B., Migliaccio, R., Thiebaut de Schotten, M., \& Bartolomeo, P. (2012). Cortical control of inhibition of return: evidence from patients with inferior parietal damage and visual neglect. Neuropsychologia, 50(5), 800-809. doi: S0028-3932(12)00030-9 [pii]

10.1016/j.neuropsychologia.2012.01.014 
Bourgeois, A., Chica, A. B., Valero-Cabre, A., \& Bartolomeo, P. (2013a). Cortical control of inhibition of return: Causal evidence for task-dependent modulations by dorsal and ventral parietal regions. Cortex, 49(8), 2229-2238. doi: S00109452(12)00326-7 [pii]

10.1016/j.cortex.2012.10.017

Bourgeois, A., Chica, A. B., Valero-Cabre, A., \& Bartolomeo, P. (2013b). Cortical control of Inhibition of Return: Exploring the causal contributions of the left parietal cortex. Cortex, 49(10), 2927-2934. doi: S0010-9452(13)00205-0 [pii]

10.1016/j.cortex.2013.08.004

Calabro, R. S., Cacciola, A., Bramanti, P., \& Milardi, D. (2015). Neural correlates of consciousness: what we know and what we have to learn! Neurological Sciences, 36(4), 505-513. doi: 10.1007/s10072-015-2072-x

Carretie, L., Rios, M., Perianez, J. A., Kessel, D., \& Alvarez-Linera, J. (2012). The role of low and high spatial frequencies in exogenous attention to biologically salient stimuli. PLoS One, 7(5), e37082. doi: 10.1371/journal.pone.0037082

Chica, A. B., Bartolomeo, P., \& Lupiáñez, J. (2013). Two cognitive and neural systems for endogenous and exogenous spatial attention. Behavioural Brain Research, 237, 107-123. doi: S0166-4328(12)00613-4 [pii] 10.1016/j.bbr.2012.09.027

Chica, A. B., Bartolomeo, P., \& Valero-Cabré, A. (2011). Dorsal and ventral parietal contributions to spatial orienting in the human brain. Journal of Neuroscience, 31(22), 8143-8149. doi: 31/22/8143 [pii]

10.1523/JNEUROSCI.5463-10.2010

Chica, A. B., Bayle, D. J., Botta, F., Bartolomeo, P., \& Paz-Alonso, P. M. (2016). Interactions between phasic alerting and consciousness in the fronto-striatal network. Scientific Reports, 6, 31868. doi: 10.1038/srep31868

Chica, A. B., Lasaponara, S., Chanes, L., Valero-Cabré, A., Doricchi, F., Lupiáñez, J., \& Bartolomeo, P. (2011). Spatial attention and conscious perception: the role of endogenous and exogenous orienting. Attention, Perception \& Psychophysics, 73(4), 1065-1081. doi: 10.3758/s13414-010-0082-6

Chica, A. B., Paz-Alonso, P. M., Valero-Cabre, A., \& Bartolomeo, P. (2013). Neural bases of the interactions between spatial attention and conscious perception. Cerebral Cortex, 23(6), 1269-1279. doi: bhs087 [pii] 10.1093/cercor/bhs087

Chica, A. B., Thiebaut de Schotten, M., Toba, M., Malhotra, P., Lupianez, J., \& Bartolomeo, P. (2011). Attention networks and their interactions after righthemisphere damage. Cortex, 48(6), 654-663. doi: S0010-9452(11)00023-2 [pii]

10.1016/j.cortex.2011.01.009

Chica, A. B., Valero-Cabré, A., Paz-Alonso, P. M., \& Bartolomeo, P. (2014). Causal contributions of the left frontal eye field to conscious perception. Cerebral Cortex, 24(3), 745-753. doi: bhs357 [pii]

$10.1093 /$ cercor/bhs357 
Ciaraffa, F., Castelli, G., Parati, E. A., Bartolomeo, P., \& Bizzi, A. (2013). Visual neglect as a disconnection syndrome? A confirmatory case report. Neurocase, 19(4), 351-359. doi: 10.1080/13554794.2012.667130

Clemens, B., Zvyagintsev, M., Sack, A. T., Heinecke, A., Willmes, K., \& Sturm, W. (2011). Revealing the functional neuroanatomy of intrinsic alertness using fMRI: methodological peculiarities. PLoS One, 6(9), e25453. doi: 10.1371/journal.pone.0025453 PONE-D-11-07567 [pii]

Corbetta, M., Patel, G., \& Shulman, G. L. (2008). The reorienting system of the human brain: from environment to theory of mind. Neuron, 58(3), 306-324.

Corbetta, M., \& Shulman, G. L. (2002). Control of goal-directed and stimulus-driven attention in the brain. Nature Reviews Neuroscience, 3(3), 201-215.

Coull, J. T., Frith, C. D., Buchel, C., \& Nobre, A. C. (2000). Orienting attention in time: behavioural and neuroanatomical distinction between exogenous and endogenous shifts. Neuropsychologia, 38(6), 808-819.

Coull, J. T., Nobre, A. C., \& Frith, C. D. (2001). The noradrenergic alpha2 agonist clonidine modulates behavioural and neuroanatomical correlates of human attentional orienting and alerting. Cereb Cortex, 11(1), 73-84.

Dale, A. M. (1999). Optimal experimental design for event-related fMRI. Hum Brain Mapp, 8(2-3), 109-114.

Dehaene, S., \& Changeux, J. P. (2011). Experimental and theoretical approaches to conscious processing. Neuron, 70(2), 200-227. doi: S0896-6273(11)00258-3 [pii] 10.1016/j.neuron.2011.03.018

Dehaene, S., Changeux, J. P., Naccache, L., Sackur, J., \& Sergent, C. (2006). Conscious, preconscious, and subliminal processing: a testable taxonomy. Trends in Cognitive Sciences, 10(5), 204-211.

Dell'Acqua, F., Scifo, P., Rizzo, G., Catani, M., Simmons, A., Scotti, G., \& Fazio, F. (2010). A modified damped Richardson-Lucy algorithm to reduce isotropic background effects in spherical deconvolution. Neuroimage, 49(2), 1446-1458. doi: 10.1016/j.neuroimage.2009.09.033

Dell'Acqua, F., Simmons, A., Williams, S. C., \& Catani, M. (2013). Can spherical deconvolution provide more information than fiber orientations? Hindrance modulated orientational anisotropy, a true-tract specific index to characterize white matter diffusion. Hum Brain Mapp, 34(10), 2464-2483. doi: $10.1002 / \mathrm{hbm} .22080$

Doricchi, F., Thiebaut de Schotten, M., Tomaiuolo, F., \& Bartolomeo, P. (2008). White matter (dis)connections and gray matter (dys)functions in visual neglect: gaining insights into the brain networks of spatial awareness. Cortex, 44(8), 983-995. 
Downar, J., Crawley, A. P., Mikulis, D. J., \& Davis, K. D. (2002). A cortical network sensitive to stimulus salience in a neutral behavioral context across multiple sensory modalities. Journal of Neurophysiology, 87(1), 615-620.

Forkel, S. J., Thiebaut de Schotten, M., Dell'Acqua, F., Kalra, L., Murphy, D. G., Williams, S. C., \& Catani, M. (2014). Anatomical predictors of aphasia recovery: a tractography study of bilateral perisylvian language networks. Brain, 137(Pt 7), 2027-2039. doi: 10.1093/brain/awu113

Ge, H., Yin, X., Xu, J., Tang, Y., Han, Y., Xu, W., . . Liu, S. (2013). Fiber pathways of attention subnetworks revealed with tract-based spatial statistics (TBSS) and probabilistic tractography. PLoS One, 8(11), e78831. doi: 10.1371/journal.pone.0078831

Kim, H. (2014). Involvement of the dorsal and ventral attention networks in oddball stimulus processing: a meta-analysis. Hum Brain Mapp, 35(5), 2265-2284. doi: $10.1002 / \mathrm{hbm} .22326$

Kincade, J. M., Abrams, R. A., Astafiev, S. V., Shulman, G. L., \& Corbetta, M. (2005). An event-related functional magnetic resonance imaging study of voluntary and stimulus-driven orienting of attention. Journal of Neuroscience, 25(18), 45934604.

Klarborg, B., Skak Madsen, K., Vestergaard, M., Skimminge, A., Jernigan, T. L., \& Baare, W. F. (2013). Sustained attention is associated with right superior longitudinal fasciculus and superior parietal white matter microstructure in children. Hum Brain Mapp, 34(12), 3216-3232. doi: 10.1002/hbm.22139

Koch, C., Massimini, M., Boly, M., \& Tononi, G. (2016). Neural correlates of consciousness: progress and problems. Nature Review Neuroscience, 17(5), 307321. doi: $10.1038 /$ nrn.2016.22

Koch, C., \& Tsuchiya, N. (2007). Attention and consciousness: two distinct brain processes. Trends in Cognitive Sciences, 11(1), 16-22.

Kusnir, F., Chica, A. B., Mitsumasu, M. A., \& Bartolomeo, P. (2011). Phasic auditory alerting improves visual conscious perception. Consciousness and Cognition, 20(4), 1201-1210. doi: S1053-8100(11)00013-4 [pii] 10.1016/j.concog.2011.01.012

Lamme, V. A. (2006). Towards a true neural stance on consciousness. Trends in Cognitive Sciences, 10(11), 494-501.

Leemans, A., \& Jones, D. K. (2009). The B-matrix must be rotated when correcting for subject motion in DTI data. Magn Reson Med, 61(6), 1336-1349.

Lunven, M., Thiebaut De Schotten, M., Bourlon, C., Duret, C., Migliaccio, R., Rode, G., \& Bartolomeo, P. (2015). White matter lesional predictors of chronic visual neglect: a longitudinal study. Brain, 138(Pt 3), 746-760. doi: 10.1093/brain/awu389 
Mack, A., \& Rock, I. (1998). Inattentional Blindness. Cambridge, MA: The MIT Press.

Manly, T., Dobler, V. B., Dodds, C. M., \& George, M. A. (2005). Rightward shift in spatial awareness with declining alertness. Neuropsychologia, 43(12), 17211728.

Marois, R., \& Ivanoff, J. (2005). Capacity limits of information processing in the brain. Trends in Cognitive Science, 9(6), 296-305. doi: S1364-6613(05)00117-8 [pii]

10.1016/j.tics.2005.04.010

Müller, H. J., \& Rabbitt, P. M. (1989). Reflexive and voluntary orienting of visual attention: Time course of activation and resistance to interruption. Journal of Experimental Psychology: Human Perception and Performance, 15(2), 315-330.

Niogi, S., Mukherjee, P., Ghajar, J., \& McCandliss, B. D. (2010). Individual Differences in Distinct Components of Attention are Linked to Anatomical Variations in Distinct White Matter Tracts. Front Neuroanat, 4, 2. doi: 10.3389/neuro.05.002.2010

Petersen, S. E., \& Posner, M. I. (2013). The attention system of the human brain: 20 years after. Annual Review Neuroscience, 35, 73-89. doi: 10.1146/annurevneuro-062111-150525

Pins, D., \& Ffytche, D. (2003). The neural correlates of conscious vision. Cerebral Cortex, 13(5), 461-474.

Posner, M. I. (1994). Attention: the mechanisms of consciousness. Proceedings of the National Academy of Sciences of the United States of America, 91(16), 73987403.

Posner, M. I. (2012). Attentional networks and consciousness. Frontiers in Psychology, 3, 64. doi: 10.3389/fpsyg.2012.00064

Rees, G., Kreiman, G., \& Koch, C. (2002). Neural correlates of consciousness in humans. Nature Reviews Neuroscience(3), 261-270.

Robertson, I. H., Mattingley, J. B., Rorden, C., \& Driver, J. (1998). Phasic alerting of neglect patients overcomes their spatial deficit in visual awareness. Nature, 395(6698), 169-172.

Robertson, I. H., Tegnér, R., Tham, K., Lo, A., \& Nimmo-Smith, I. (1995). Sustained attention training for unilateral neglect: Theoretical and rehabilitation implications. Journal of Clinical and Experimental Neuropsychology, 17(3), 416-430.

Rojkova, K., Volle, E., Urbanski, M., Humbert, F., Dell'Acqua, F., \& Thiebaut de Schotten, M. (2016). Atlasing the frontal lobe connections and their variability due to age and education: a spherical deconvolution tractography study. Brain Struct Funct, 221(3), 1751-1766. doi: 10.1007/s00429-015-1001-3 
Seth, A. K. (2009). Explanatory correlates of consciousness: Theoretical and computational challenges. Cognitive Computation, 1, 50-63.

Shinoura, N., Suzuki, Y., Yamada, R., Tabei, Y., Saito, K., \& Yagi, K. (2009). Damage to the right superior longitudinal fasciculus in the inferior parietal lobe plays a role in spatial neglect. Neuropsychologia, 47(12), 2600-2603. doi: 10.1016/j.neuropsychologia.2009.05.010

Shulman, G. L., Astafiev, S. V., Franke, D., Pope, D. L., Snyder, A. Z., McAvoy, M. P., \& Corbetta, M. (2009). Interaction of stimulus-driven reorienting and expectation in ventral and dorsal frontoparietal and basal ganglia-cortical networks. Journal of Neuroscience, 29(14), 4392-4407. doi: 29/14/4392 [pii]

10.1523/JNEUROSCI.5609-08.2009

Shulman, G. L., Astafiev, S. V., McAvoy, M. P., d'Avossa, G., \& Corbetta, M. (2007). Right TPJ deactivation during visual search: Functional significance and support for a filter hypothesis. Cerebral Cortex, 1-9.

Sperling, G. (1960). The information available in brief visual presentations. Psychological Monographs, 74(11), 1-29.

Sturm, W., de Simone, A., Krause, B. J., Specht, K., Hesselmann, V., Radermacher, I., . . . Willmes, K. (1999). Functional anatomy of intrinsic alertness: evidence for a fronto-parietal-thalamic-brainstem network in the right hemisphere. Neuropsychologia, 37(7), 797-805. doi: S0028393298001419 [pii]

Sturm, W., Longoni, F., Fimm, B., Dietrich, T., Weis, S., Kemna, S., . . Willmes, K. (2004). Network for auditory intrinsic alertness: a PET study. Neuropsychologia, 42(5), 563-568. doi: S0028393203002835 [pii]

Sturm, W., \& Willmes, K. (2001). On the functional neuroanatomy of intrinsic and phasic alertness. Neuroimage, 14(1 Pt 2), S76-84. doi: 10.1006/nimg.2001.0839 S1053-8119(01)90839-0 [pii]

Thiebaut de Schotten, M., Dell'Acqua, F., Forkel, S. J., Simmons, A., Vergani, F., Murphy, D. G., \& Catani, M. (2011). A lateralized brain network for visuospatial attention. Nat Neurosci, 14(10), 1245-1246. doi: nn.2905 [pii]

$10.1038 / \mathrm{nn} .2905$

Thiebaut de Schotten, M., Tomaiuolo, F., Aiello, M., Merola, S., Silvetti, M., Lecce, F., ... Doricchi, F. (2014). Damage to white matter pathways in subacute and chronic spatial neglect: a group study and 2 single-case studies with complete virtual "in vivo" tractography dissection. Cerebral Cortex, 24(3), 691-706. doi: bhs351 [pii] 10.1093/cercor/bhs351

Thiebaut de Schotten, M., Urbanski, M., Duffau, H., Volle, E., Lévy, R., Dubois, B., \& Bartolomeo, P. (2005). Direct evidence for a parietal-frontal pathway subserving spatial awareness in humans. Science, 5744, 2226-2228. 
Thiel, C. M., \& Fink, G. R. (2007). Visual and auditory alertness: modality-specific and supramodal neural mechanisms and their modulation by nicotine. Journal of Neurophysiology, 97(4), 2758-2768. doi: 10.1152/jn.00017.2007

Thimm, M., Fink, G. R., Kust, J., Karbe, H., \& Sturm, W. (2006). Impact of alertness training on spatial neglect: a behavioural and fMRI study. Neuropsychologia, 44(7), 1230-1246.

Tsuchiya, N., Wilke, M., Frassle, S., \& Lamme, V. A. (2015). No-Report Paradigms: Extracting the True Neural Correlates of Consciousness. Trends in Cognitive Sciences, 19(12), 757-770. doi: 10.1016/j.tics.2015.10.002

Uddin, L. Q. (2015). Salience processing and insular cortical function and dysfunction. Nature Review Neuroscience, 16(1), 55-61. doi: 10.1038/nrn3857

Urbanski, M., Thiebaut de Schotten, M., Rodrigo, S., Catani, M., Oppenheim, C., Touze, E., . . . Bartolomeo, P. (2008). Brain networks of spatial awareness: Evidence from diffusion tensor imaging tractography. Journal of Neurology, Neurosurgery and Psychiatry, 79(5), 598-601.

Urbanski, M., Thiebaut de Schotten, M., Rodrigo, S., Oppenheim, C., Touze, E., Meder, J. F., . . Bartolomeo, P. (2011). DTI-MR tractography of white matter damage in stroke patients with neglect. Experimental Brain Research, 208(4), 491-505. doi: 10.1007/s00221-010-2496-8

Wingen, M., Kuypers, K. P., van de Ven, V., Formisano, E., \& Ramaekers, J. G. (2008). Sustained attention and serotonin: a pharmaco-fMRI study. Hum Psychopharmacol, 23(3), 221-230. doi: 10.1002/hup.923

Wyart, V., Dehaene, S., \& Tallon-Baudry, C. (2011). Early dissociation between neural signatures of endogenous spatial attention and perceptual awareness during visual masking. Frontiers in Human Neuroscience, 6, 16. doi: 10.3389/fnhum.2012.00016

Wyart, V., \& Tallon-Baudry, C. (2008). Neural dissociation between visual awareness and spatial attention. Journal of Neuroscience, 28(10), 2667-2679. doi: doi: 10.1523/JNEUROSCI.4748-07.2008.

Yanaka, H. T., Saito, D. N., Uchiyama, Y., \& Sadato, N. (2010). Neural substrates of phasic alertness: a functional magnetic resonance imaging study. Neuroscience Research, 68(1), 51-58. doi: S0168-0102(10)00142-2 [pii] 10.1016/j.neures.2010.05.005

Zeki, S. (2005). The Ferrier Lecture 1995 behind the seen: the functional specialization of the brain in space and time. Philos Trans R Soc Lond B Biol Sci, 360(1458), 1145-1183. doi: 10.1098/rstb.2005.1666 
Table 1. Significant predictors of the functional interaction in the left FEF for the classical lineal regression, using the backward method, in the exogenous orienting study.

Model Summary

\begin{tabular}{rrrrr}
\hline Model & R & \multicolumn{1}{c}{$\mathbf{R}^{\mathbf{2}}$} & Adjusted $\mathbf{R}^{\mathbf{2}}$ & RMSE \\
\hline 1 & 0.837 & 0.701 & 0.592 & 0.639 \\
2 & 0.798 & 0.636 & 0.545 & 0.674
\end{tabular}

\begin{tabular}{|c|c|c|c|c|c|c|}
\hline \multicolumn{7}{|l|}{$\overline{\text { ANOVA }}$} \\
\hline Model & & Sum of Squares & df & Mean Square & $\mathbf{F}$ & $\mathbf{p}$ \\
\hline \multirow[t]{3}{*}{1} & Regression & 10.508 & 4 & 2.627 & 6.434 & 0.006 \\
\hline & Residual & 4.492 & 11 & 0.408 & & \\
\hline & Total & 15.000 & 15 & & & \\
\hline \multirow[t]{3}{*}{2} & Regression & 9.544 & 3 & 3.181 & 6.997 & 0.006 \\
\hline & Residual & 5.456 & 12 & 0.455 & & \\
\hline & Total & 15.000 & 15 & & & \\
\hline
\end{tabular}

\begin{tabular}{|c|c|c|c|c|c|c|c|}
\hline \multicolumn{8}{|c|}{ Coefficients } \\
\hline \multirow[b]{2}{*}{ Model } & & \multirow[b]{2}{*}{ Unstandardized } & \multirow[b]{2}{*}{$\begin{array}{c}\text { Standard } \\
\text { Error }\end{array}$} & \multirow[b]{2}{*}{ Standardized } & \multirow[b]{2}{*}{$\mathbf{p}$} & \multicolumn{2}{|c|}{ Collinearity Statistic } \\
\hline & & & & & & Tolerance & VIF \\
\hline 1 & intercept & -0.000 & 0.160 & & $-3.815 \mathrm{e}-61.000$ & & \\
\hline \multirow{8}{*}{2} & Right SLF III & 0.279 & 0.182 & 0.279 & 1.5370 .153 & 0.824 & 1.214 \\
\hline & Left SLF III & -0.511 & 0.171 & -0.511 & $\begin{array}{lll}-2.981 & 0.012\end{array}$ & 0.928 & 1.078 \\
\hline & $\begin{array}{l}\text { Behavioral } \\
\text { index }\end{array}$ & -0.363 & 0.171 & -0.363 & -2.1180 .058 & 0.929 & 1.077 \\
\hline & Right IPS & 0.613 & 0.182 & 0.613 & 3.3750 .006 & 0.826 & 1.210 \\
\hline & intercept & -0.000 & 0.169 & & $-3.311 \mathrm{e}-61.000$ & & \\
\hline & Left SLF III & -0.451 & 0.176 & -0.451 & -2.5620 .025 & 0.978 & 1.023 \\
\hline & $\begin{array}{l}\text { Behavioral } \\
\text { index }\end{array}$ & -0.383 & 0.180 & -0.383 & -2.1260 .055 & 0.934 & 1.070 \\
\hline & Right IPS & 0.510 & 0.178 & 0.510 & 2.8640 .014 & 0.955 & 1.047 \\
\hline
\end{tabular}


Table 2. Control analysis using the integrity of the SLF I as predictor in the exogenous orienting study.

Model Summary

\begin{tabular}{rrrrr}
\hline Model & R & $\mathbf{R}^{\mathbf{2}}$ & Adjusted R $^{\mathbf{2}}$ & RMSE \\
\hline 1 & 0.773 & 0.598 & 0.451 & 0.741 \\
2 & 0.703 & 0.494 & 0.368 & 0.795 \\
3 & 0.661 & 0.437 & 0.351 & 0.806 \\
4 & 0.585 & 0.343 & 0.296 & 0.839
\end{tabular}

\begin{tabular}{|c|c|c|c|c|c|c|}
\hline \multicolumn{7}{|c|}{$\overline{\text { ANOVA }}$} \\
\hline Model & & Sum of Squares & df & Mean Square & $\mathbf{F}$ & p \\
\hline \multirow[t]{3}{*}{1} & Regression & 8.964 & 4 & 2.241 & 4.084 & 0.029 \\
\hline & Residual & 6.036 & 11 & 0.549 & & \\
\hline & Total & 15.000 & 15 & & & \\
\hline \multirow[t]{3}{*}{2} & Regression & 7.410 & 3 & 2.470 & 3.906 & 0.037 \\
\hline & Residual & 7.590 & 12 & 0.632 & & \\
\hline & Total & 15.000 & 15 & & & \\
\hline \multirow[t]{3}{*}{3} & Regression & 6.559 & 2 & 3.280 & 5.051 & 0.024 \\
\hline & Residual & 8.441 & 13 & 0.649 & & \\
\hline & Total & 15.000 & 15 & & & \\
\hline \multirow[t]{3}{*}{4} & Regression & 5.141 & 1 & 5.141 & 7.300 & 0.017 \\
\hline & Residual & 9.859 & 14 & 0.704 & & \\
\hline & Total & 15.000 & 15 & & & \\
\hline
\end{tabular}

\begin{tabular}{|c|c|c|c|c|c|c|c|}
\hline \multicolumn{8}{|c|}{ Coefficients } \\
\hline \multirow[b]{2}{*}{ Model } & & \multirow[b]{2}{*}{ Unstandardized } & \multirow[b]{2}{*}{$\begin{array}{l}\text { Standard } \\
\text { Error }\end{array}$} & \multirow[b]{2}{*}{ Standardized } & \multirow[b]{2}{*}{$\mathbf{p}$} & \multicolumn{2}{|c|}{ Collinearity Statistics } \\
\hline & & & & & & Tolerance & VIF \\
\hline 1 & intercept & -0.000 & 0.185 & & $-4.062 \mathrm{e}-61.000$ & & \\
\hline & $\begin{array}{l}\text { Behavioral } \\
\text { index }\end{array}$ & -0.409 & 0.202 & -0.409 & -2.0230 .068 & 0.896 & 1.116 \\
\hline & Right IPS & 0.662 & 0.212 & 0.662 & 3.1270 .010 & 0.817 & 1.224 \\
\hline & Left SLF I & -0.544 & 0.270 & -0.544 & -2.0150 .069 & 0.502 & 1.992 \\
\hline & Right SLF I & 0.411 & 0.244 & 0.411 & 1.6830 .121 & 0.614 & 1.628 \\
\hline 2 & intercept & -0.000 & 0.199 & & $-3.925 e-61.000$ & & \\
\hline & $\begin{array}{l}\text { Behavioral } \\
\text { index }\end{array}$ & -0.370 & 0.216 & -0.370 & -1.7180 .111 & 0.908 & 1.102 \\
\hline & Right IPS & 0.612 & 0.225 & 0.612 & 2.7220 .019 & 0.833 & 1.200 \\
\hline & Left SLF I & -0.266 & 0.229 & -0.266 & $\begin{array}{lll}-1.160 & 0.269\end{array}$ & 0.802 & 1.247 \\
\hline 3 & intercept & -0.000 & 0.201 & & $-2.586 e-61.000$ & & \\
\hline & $\begin{array}{l}\text { Behavioral } \\
\text { index }\end{array}$ & -0.315 & 0.213 & -0.315 & -1.4780 .163 & 0.955 & 1.047 \\
\hline & Right IPS & 0.519 & 0.213 & 0.519 & 2.4380 .030 & 0.955 & 1.047 \\
\hline 4 & intercept & -0.000 & 0.210 & & $-1.744 \mathrm{e}-61.000$ & & \\
\hline & Right IPS & 0.585 & 0.217 & 0.585 & 2.7020 .017 & 1.000 & 1.000 \\
\hline
\end{tabular}


Table 3. Significant predictors of the functional interaction in the left ACC for the classical lineal regression, using the backward method, in the phasic alerting study.

Model Summary

\begin{tabular}{rrrrr}
\hline Model & R & $\mathbf{R}^{2}$ & Adjusted R & RMSE \\
\hline 1 & 0.722 & 0.521 & 0.362 & 0.799 \\
2 & 0.719 & 0.517 & 0.405 & 0.771 \\
3 & 0.715 & 0.512 & 0.442 & 0.747
\end{tabular}

\begin{tabular}{|c|c|c|c|c|c|c|}
\hline \multicolumn{7}{|l|}{ ANOVA } \\
\hline Model & & Sum of Squares & df & Mean Square & $\mathbf{F}$ & $\mathbf{p}$ \\
\hline \multirow[t]{3}{*}{1} & Regression & 8.341 & 4 & 2.085 & 3.267 & 0.050 \\
\hline & Residual & 7.659 & 12 & 0.638 & & \\
\hline & Total & 16.000 & 16 & & & \\
\hline \multirow[t]{3}{*}{2} & Regression & 8.269 & 3 & 2.756 & 4.634 & 0.020 \\
\hline & Residual & 7.731 & 13 & 0.595 & & \\
\hline & Total & 16.000 & 16 & & & \\
\hline \multirow[t]{3}{*}{3} & Regression & 8.191 & 2 & 4.095 & 7.342 & 0.007 \\
\hline & Residual & 7.809 & 14 & 0.558 & & \\
\hline & Total & 16.000 & 16 & & & \\
\hline
\end{tabular}

\begin{tabular}{|c|c|c|c|c|c|c|c|c|}
\hline \multicolumn{9}{|c|}{ Coefficients } \\
\hline \multirow[b]{2}{*}{ Model } & & \multirow[b]{2}{*}{ Unstandardized } & \multirow[b]{2}{*}{$\begin{array}{c}\text { Standard } \\
\text { Error }\end{array}$} & \multirow[b]{2}{*}{ Standardized } & \multirow[b]{2}{*}{ t } & \multirow[b]{2}{*}{$\mathbf{p}$} & \multicolumn{2}{|c|}{ Collinearity Statistics } \\
\hline & & & & & & & Tolerance & VIF \\
\hline 1 & intercept & -0.078 & 0.196 & & -0.400 & 0.696 & & \\
\hline & Right SLF III & 0.076 & 0.227 & 0.076 & 0.337 & 0.742 & 0.774 & 1.292 \\
\hline & Left SLF III & 0.417 & 0.227 & 0.417 & 1.840 & 0.091 & 0.775 & 1.290 \\
\hline & $\begin{array}{l}\text { Behavioral } \\
\text { index }\end{array}$ & 0.088 & 0.212 & 0.088 & 0.415 & 0.685 & 0.885 & 1.130 \\
\hline & Right Caudate & 0.608 & 0.231 & 0.540 & 2.631 & 0.022 & 0.948 & 1.055 \\
\hline 2 & intercept & -0.079 & 0.189 & & -0.418 & 0.683 & & \\
\hline & Left SLF III & 0.452 & 0.196 & 0.452 & 2.308 & 0.038 & 0.971 & 1.030 \\
\hline & $\begin{array}{l}\text { Behavioral } \\
\text { index }\end{array}$ & 0.072 & 0.200 & 0.072 & 0.362 & 0.723 & 0.931 & 1.074 \\
\hline & Right Caudate & 0.613 & 0.223 & 0.544 & 2.756 & 0.016 & 0.952 & 1.050 \\
\hline 3 & intercept & -0.077 & 0.183 & & -0.419 & 0.681 & & \\
\hline & Left SLF III & 0.463 & 0.187 & 0.463 & 2.480 & 0.026 & 0.999 & 1.001 \\
\hline & Right Caudate & 0.596 & 0.210 & 0.529 & 2.831 & 0.013 & 0.999 & 1.001 \\
\hline
\end{tabular}


Table 4. Control analysis using the integrity of the SLF I as predictor in the phasic alerting study.

Model Summary

\begin{tabular}{rrrrr}
\hline Model & R & $\mathbf{R}^{\mathbf{2}}$ & Adjusted R & RMSE \\
\hline 1 & 0.635 & 0.404 & 0.205 & 0.892 \\
2 & 0.627 & 0.393 & 0.253 & 0.864 \\
3 & 0.605 & 0.367 & 0.276 & 0.851 \\
4 & 0.546 & 0.298 & 0.251 & 0.866
\end{tabular}

\begin{tabular}{|c|c|c|c|c|c|c|}
\hline \multicolumn{7}{|l|}{$\overline{\text { ANOVA }}$} \\
\hline Model & & Sum of Squares & df & Mean Square & $\mathbf{F}$ & $\mathbf{p}$ \\
\hline \multirow[t]{3}{*}{1} & Regression & 6.458 & 4 & 1.615 & 2.031 & 0.154 \\
\hline & Residual & 9.542 & 12 & 0.795 & & \\
\hline & Total & 16.000 & 16 & & & \\
\hline \multirow[t]{3}{*}{2} & Regression & 6.286 & 3 & 2.095 & 2.804 & 0.081 \\
\hline & Residual & 9.714 & 13 & 0.747 & & \\
\hline & Total & 16.000 & 16 & & & \\
\hline \multirow[t]{3}{*}{3} & Regression & 5.865 & 2 & 2.932 & 4.051 & 0.041 \\
\hline & Residual & 10.135 & 14 & 0.724 & & \\
\hline & Total & 16.000 & 16 & & & \\
\hline \multirow[t]{3}{*}{4} & Regression & 4.761 & 1 & 4.761 & 6.355 & 0.024 \\
\hline & Residual & 11.239 & 15 & 0.749 & & \\
\hline & Total & 16.000 & 16 & & & \\
\hline
\end{tabular}

\begin{tabular}{|c|c|c|c|c|c|c|c|c|}
\hline \multicolumn{9}{|c|}{ Coefficients } \\
\hline \multirow{2}{*}{\multicolumn{2}{|c|}{ Model }} & \multirow[b]{2}{*}{ Unstandardized } & \multirow[b]{2}{*}{$\begin{array}{c}\text { Standard } \\
\text { Error }\end{array}$} & \multirow[b]{2}{*}{ Standardized } & \multirow[b]{2}{*}{$\mathbf{t}$} & \multirow[b]{2}{*}{$\mathbf{p}$} & \multicolumn{2}{|c|}{ Collinearity Statistics } \\
\hline & & & & & & & Tolerance & VIF \\
\hline \multirow[t]{5}{*}{1} & intercept & -0.075 & 0.219 & & -0.343 & 0.738 & & \\
\hline & Left SLF I & -0.237 & 0.371 & -0.237 & -0.639 & 0.535 & 0.361 & 2.771 \\
\hline & Right SLF I & 0.430 & 0.354 & 0.430 & 1.216 & 0.248 & 0.397 & 2.518 \\
\hline & $\begin{array}{l}\text { Behavioral } \\
\text { index }\end{array}$ & 0.108 & 0.232 & 0.108 & 0.465 & 0.650 & 0.926 & 1.080 \\
\hline & Right Caudate & 0.584 & 0.284 & 0.518 & 2.056 & 0.062 & 0.783 & 1.278 \\
\hline \multirow[t]{4}{*}{2} & intercept & -0.071 & 0.212 & & -0.334 & 0.744 & & \\
\hline & Left SLF I & -0.266 & 0.355 & -0.266 & -0.751 & 0.466 & 0.372 & 2.692 \\
\hline & Right SLF I & 0.458 & 0.338 & 0.458 & 1.355 & 0.198 & 0.409 & 2.446 \\
\hline & Right Caudate & 0.550 & 0.266 & 0.488 & 2.066 & 0.059 & 0.837 & 1.195 \\
\hline \multirow[t]{3}{*}{3} & intercept & -0.081 & 0.209 & & -0.389 & 0.703 & & \\
\hline & Right SLF I & 0.263 & 0.213 & 0.263 & 1.235 & 0.237 & 0.997 & 1.003 \\
\hline & Right Caudate & 0.630 & 0.240 & 0.559 & 2.626 & 0.020 & 0.997 & 1.003 \\
\hline \multirow[t]{2}{*}{4} & intercept & -0.079 & 0.212 & & -0.373 & 0.714 & & \\
\hline & Right Caudate & 0.615 & 0.244 & 0.546 & 2.521 & 0.024 & 1.000 & 1.000 \\
\hline
\end{tabular}


(A) Exogenous orienting experiment

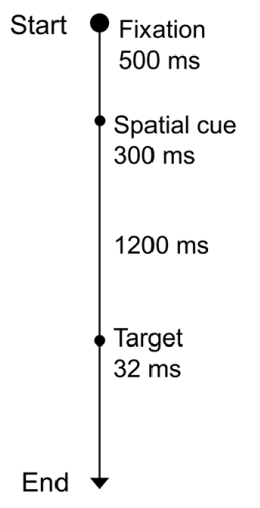

(B) Phasic alerting experiment
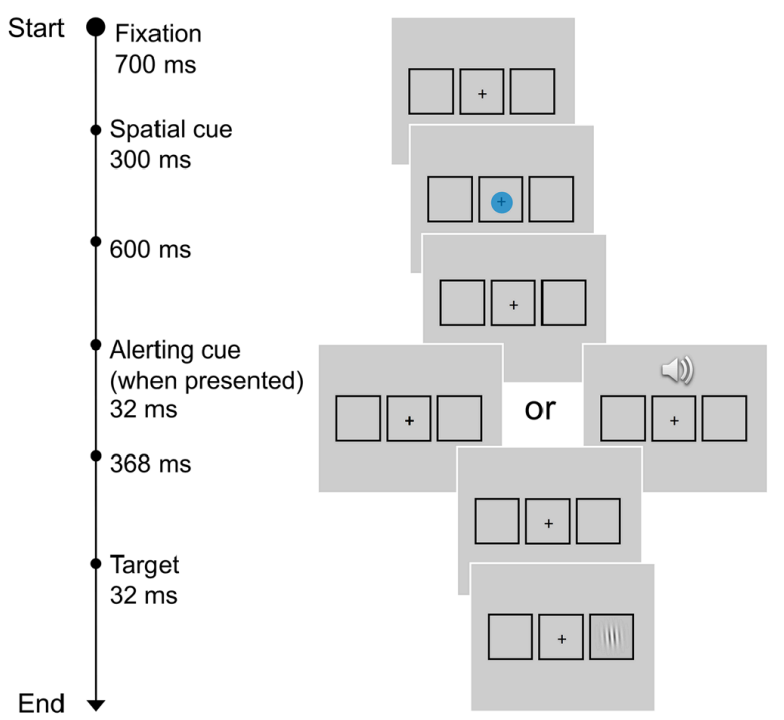

Figure 1. Experimental task used in the (A) exogenous orienting and (B) phasic alerting studies. In the latter, a central symbolic cue was also used to manipulate endogenous orienting, but it only demonstrated a weak interaction with consciousness in the thalamus. 
(A)
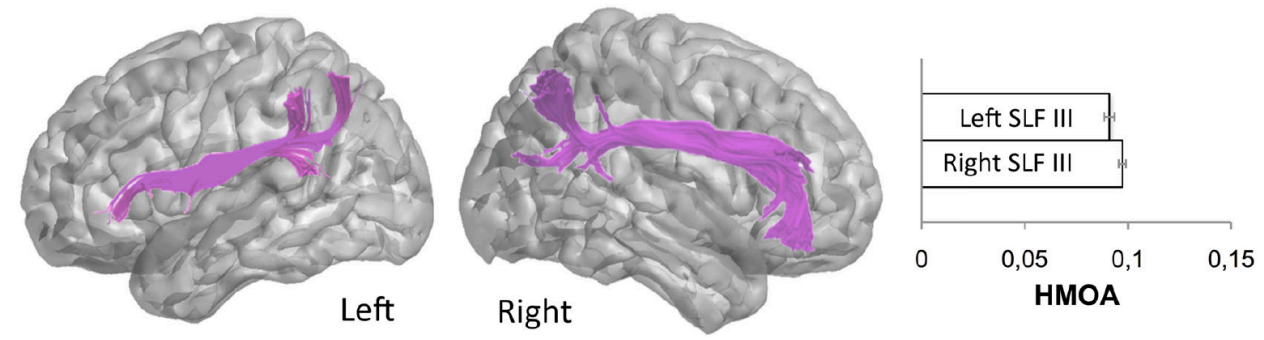

(B)
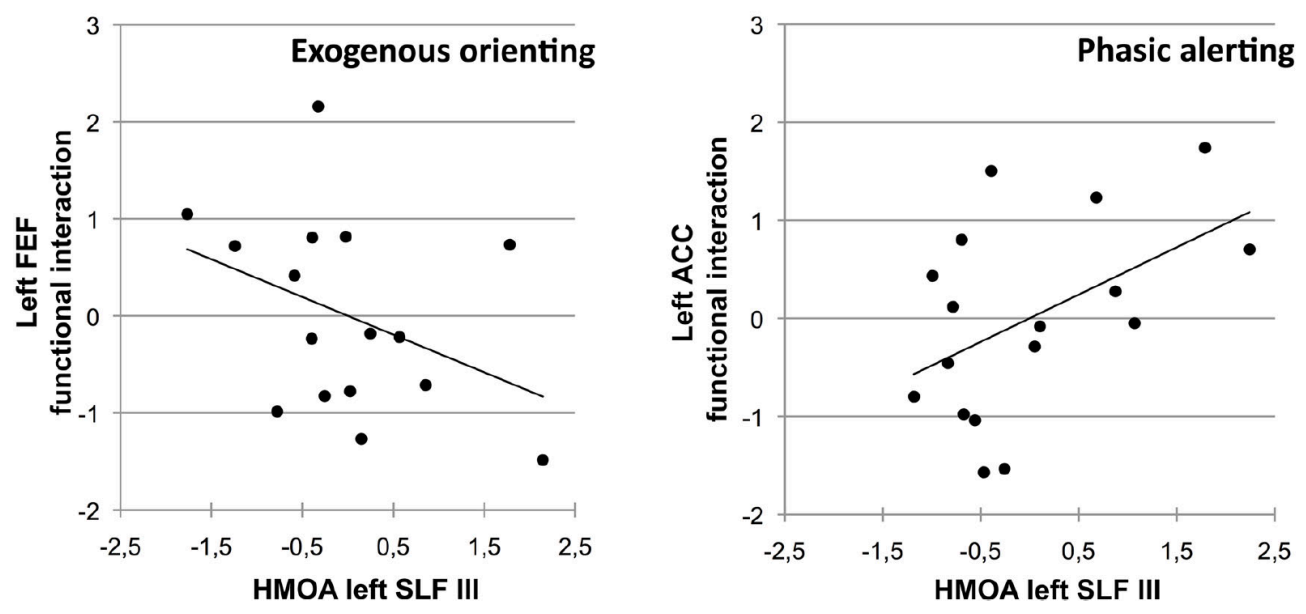

Figure 2. A) Graphical representation of the SLF III in the left and right hemispheres. The graph on the right represents the mean HMOA values in each hemisphere (nonnormalized data). B) Correlation between the functional interaction between exogenous orienting and consciousness measured in left FEF and the HMOA of the left SLF III (Left Panel), and between phasic alerting and consciousness measured in the left ACC and the HMOA of the left SLF III (Right Panel). Normalized data by Z score transformation. 